\title{
Reverse Auction Based Green Offloading Scheme for Small Cell Heterogeneous Networks
}

\author{
Xiaodong Xu, Chunjing Yuan, Jianhui Li, Huixin Zhang, and Xiaofeng Tao \\ National Engineering Laboratory for Mobile Network Security, Beijing University of Posts and Telecommunications, Beijing, China \\ Correspondence should be addressed to Xiaodong Xu; xuxiaodong@bupt.edu.cn
}

Received 1 February 2016; Accepted 17 July 2016

Academic Editor: Gabriel-Miro Muntean

Copyright ( 2016 Xiaodong Xu et al. This is an open access article distributed under the Creative Commons Attribution License, which permits unrestricted use, distribution, and reproduction in any medium, provided the original work is properly cited.

\begin{abstract}
The small cell is treated as a promising proposition to provide hot spot capacity and higher data rates. However, even with dense small cell deployment scenario, the heavy traffic load pressure and low energy efficiency in the small cell heterogeneous network (HetNet) still exist. Therefore, how to make the best use of densely deployed small cells under HetNet environments becomes the focus of researches. Offloading provides a feasible solution to promote cooperation between macrocells and small cells for user traffic supporting. In this paper, we propose the reverse auction based Green Offloading (GO) scheme for energy efficiency improvements. The proposed GO scheme employs the reverse auction theory to handle the offloading decision process, aiming at maximizing the system energy efficiency under the constraints of user Quality of Service (QoS) requirements, bandwidth, and transmission power limitations. Moreover, the reverse auction model gives the facility of multicell coordination transmissions with multiple winners situation. The energy efficiency optimization problem with constraints is solved by Dynamic Programming method with Karush-Kuhn-Tucker (KKT) conditions. The Individual Rationality and Truthfulness of the reverse auction model are also proved. By comparing the energy efficiency performances of the proposed GO scheme with current works within the Long Term Evolution-Advanced (LTE-A) system downlink scenario, simulation results show the improvements of the proposed GO scheme.
\end{abstract}

\section{Introduction}

With the rapid development of wireless communications, there is a growing conflict between increasing mobile traffic demands and limited radio resources. Many projects predict an over 500-fold growth on mobile data traffic in 10 years (2010-2020) [1]. In order to satisfy this tremendous demand, lots of small cells have been deployed in both indoor and outdoor environments for hot-spot capacity improvements [2]. Moreover, the corresponding research and standard work for the 5th Generation Mobile Communication System (5G) are in a full swing $[3,4]$. For future $5 \mathrm{G}$ networks, the small cell heterogeneous network (HetNet) is believed as an important solution to fulfill the capacity booming requirements.

The deployment of the small cell HetNet offers enhanced capacity and expansile coverage and alleviates the heavy traffic burden in macrocells. Relying on above advantages, mobile operators all over the world are now keen on small cell HetNet deployments. According to statistics, the total number of already-deployed small cells has exceeded that of macrocells [5]. Despite the fact that lots of small cells have been deployed densely, simply relying on increasing the spatial density of small cells is not an economically sustainable manner to fulfill the growing demands. Because the deployment of a new Base Station (BS) goes along with large expenses like energy consumptions, laying of dedicated backhaul, and network replanning processes, therefore, how to take full advantages of the already-deployed macrocell and small cell BSs becomes the key issue for the mobile industry. In order to solve this problem, researches have been focusing on the offloading strategy gradually, which is believed as a promising paradigm to improve the network utilizing for densely deployed small cell HetNets [6, 7].

Offloading is a kind of technology that transfers the traffic load from one network to another under certain conditions, typically in case of heavy traffic burden or low efficiency networks. One typical traditional scenario for offloading application is about the cellular network and Wireless Fidelity (WiFi) network. Through offloading the cellular traffic to WiFi networks, the load pressure of the cellular network 
could be relieved [8]. In recent years, some researches have been conducted to refine the offloading scheme within cellular networks to alleviate the increasing traffic burden of macrocells. Authors of [9] analyze the proportion of traffic that can be offloaded from macrocells to femtocells to increase the system capacity. The offloading gain for the macrocell and femtocell colocated HetNets is explored in [10]. Except for enhancing capacity purposes, the energy efficiency metric is also considered during offloading processes. In [11], the system energy efficiency is improved through offloading the high Quality of Service (QoS) requirement traffic to femtocells. But the fairness between different users is neglected. In [12], authors verify the offloading gain in the macrocell and femtocell orthogonal frequency division multiple access system by analytical evaluations. Nevertheless, the transmission power allocation is not taken into account. Benefitting from the information exchange between macrocells and picocells, a traffic offloading based on fractional frequency reuse (TOFFR) algorithm is proposed to improve the system energy efficiency in [13]. Authors in [14] propose a traffic offloading scheme based on reverse auction mechanism and greedy algorithms, which incentivizes femtocell owners to rent their underutilized spectrum for the enhancement of network performances in HetNets.

From the analyses of above researches, there are several methods adopted in offloading processes. The stochastic geometry is adopted to analyze the statistical status of the offloading gain [9]. Game theory approaches are explored by offloading researches in $[15,16]$. Furthermore, the auction theory is also employed to support the offloading target decision and network performance improvements $[10,14,17$, 18]. The forward auction model is applied in [10] for the offloading between different operators. References [14, 18] employ the reverse auction model to establish the offloading procedures; the properties of the proposed models are also analyzed. We also applied the forward auction model to improve the system energy efficiency of offloading in the HetNet [17].

In economics, auction is a common means to determine the value of an item which has a valuable price. Nowadays, the auction theory has been widely used in various fields, such as the dynamic spectrum management in cognitive networks [19] and hybrid access in HetNets [20]. The auction model is particularly suitable for the processes that need information exchanges and target choosing with pricing issues. Most of the auction models employed currently are forward auctions, which typically involve a single seller and multiple buyers. The buyers send bids to compete for the item sold by the seller. The forward auction model is popular in dealing with the decisions among multiple operators to get minimum costs [10], while the reverse auction model could be used in the situation that one buyer wants to offer reasonable prices to get service from multiple sellers [21]. The offloading process is just adapted to the reverse auction applications that the offloading user is treated as the buyer, while the candidate BSs are acting as the sellers. Therefore, in this paper, a reverse auction process with first price sealed bid mechanism is adopted in the offloading research. The reverse auction model involves a single buyer and multiple sellers, where the buyer makes the decision on whose bid will be accepted according to the bids sent by multiple sellers.

For the offloading process researched in small cell HetNets, there are two main concerns adapted to the reverse auction model:

(1) The offloading user needs to select the target BS, which is modeled as one "buyer" with multicandidate "sellers." The offloading user is acting as a buyer to get service from multicandidate BSs as sellers. The candidate BSs offer their bids to compete for the serving opportunity of the offloading user, which could improve their performances such as throughputs or resource/energy efficiency.

(2) The aforementioned "bid" offered by the candidate BSs could be their available subcarriers and transmission power. The "buyer" needs to pay with the price determined by the reverse auction for getting the service, where the price is usually defined as the opportunity cost of the offloading target decision.

In order to improve the system energy efficiency in the dense small cell HetNet deployment scenario, we propose a Green Offloading (GO) scheme through the Vickrey-ClarkeGrove (VCG) based reverse auction model [22]. The small cells will be incentivized to help offloading the traffic from macrocells, which allows the operator to express diverse preferences for densely deployed HetNets. The properties of the reverse auction model, such as the IndividualRationality and Truthfulness, are also proved in this paper to guarantee the optimality of the proposed reverse auction mechanism.

In this paper, the employed reverse auction model with first price sealed bid mechanism is formulated for the offloading process. The energy efficiency optimization problem within the offloading process is modeled with constraints of user guaranteed QoS requirements. The optimization problem is solved by the Karush-Kuhn-Tucker (KKT) conditions and Dynamic Programming method. The system-level simulation evaluations are conducted for the proposed reverse auction based GO scheme with taking current schemes [13, $14]$ as comparisons.

The contributions of this paper include three aspects:

(1) The reverse auction model is explored in the offloading scheme design with the first price sealed bid mechanism, which matches the mobility based offloading process well with limited negotiations and delays.

(2) The multiple winning bidders are also supported for BS coordination transmissions.

(3) The optimization of the system energy efficiency improvement is solved with guaranteed user throughput in the offloading target decision and reallocation of both the resource block and transmission power.

The rest of this paper is organized as the following. In Section 2, the system model is described and the reverse auction model applied in this paper is clarified. The reverse 
auction based GO scheme for small cell HetNets is proposed in Section 3. In Section 4, the simulation results are presented and discussed with comparison schemes. Finally, concluding remarks are drawn in Section 5.

\section{System Model}

In this section, the system model of the proposed reverse auction based GO scheme is formulated. The network deployment scenario will be introduced at first and the notation definitions about the reverse auction model based offloading process are described afterwards.

2.1. Deployment Scenario. We focus on the small cell and macrocell overlaid HetNet deployment scenario in this paper. According to the 3rd Generation Partnership Project (3GPP) Long Term Evolution-Advanced (LTE-A) standards, due to the scarcity of available spectrum, the macrocell tier and small cell tier will be inevitably deployed with the shared spectrum manner. In this paper, we will focus on the shared spectrum scenario with considerations of cross-tier interferences, which is more realistic. Furthermore, the coordination technologies, such as the Coordinated Multipoint Transmission/Reception (CoMP) defined by 3GPP [23], are supported in above small cell HetNet deployment scenario. CoMP will bring benefits to the cell edge throughput and energy efficiency, which helps the offloading users usually located in the cell edge area.

In order to address the dense deployment scenario for future heavy-traffic requirements, each macrocell coverage area is covered by several small cell clusters, the number of which will be 1, 4, or 10 based on 3GPP simulation methodology [24]. Each small cell cluster includes several small cell BSs, the number of which will be 4 or 10 [25]. Both small cell clusters and small cell BSs are considerably less planed as opposed to the typical planned macrocell deployments.

Based on the standard discussions on the small cell HetNet deployments in 3GPP [26], the macrocell will take charge of the control plane for both the macrocell and colocated small cells within its coverage. The main responsibility of the small cell tier is to offload the high data-rate service from the macrocell, while the macrocell tier handles the low datarate traffic and high mobility users. This is the typical actual deployment demand for the HetNet. The traffic model of the user is File Transfer Protocol (FTP) Model 1 as full-buffer [23]. The system energy efficiency will be the optimization objective during the offloading processes with constraints of user QoS guaranteeing.

The handover solution for the offloading operation is different from the standard handover criterion due to the user mobility. For small cell HetNets, 3GPP also discussed new handover criterion for macro-to-small cells with Cell Range Extension (CRE), which is defined by 3GPP standard [27]. Assisted by the CRE, the downlink Reference Signal Received Power (RSRP) of small cells could be added with a bias. By this means, the user will be encouraged to do handover to small cells, which will help to implement the handover for offloading users or load balancing operations.

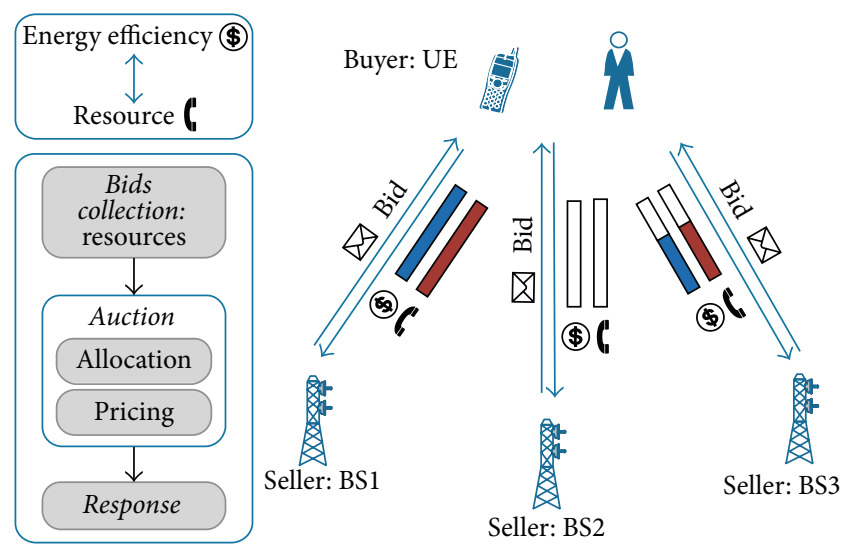

FIgURE 1: The framework of reverse auction based GO scheme.

2.2. Reverse Auction. One typical implementation scenario of GO scheme is given in this section. Considering the users' immense offloading potential in dense small cell HetNet, a reverse auction model based offloading scheme is suitable to motivate users to conduct traffic offloading that could achieve higher system energy efficiency. Figure 1 illustrates the typical scenario of offloading process with reverse auction model. According to the reverse auction model, the User Equipment (UE) acts as the buyer, who ensures higher system energy efficiency in exchange of bandwidth and transmission power resources provided by the BS to serve the offloading UE. When the UE requests data transmission, its current serving BS is encouraged to broadcast the offloading request to all of its neighboring BSs. All the BSs receiving the request will send their bids along with their available resources to the UE's current serving BS. The information included in each bid contains the amount of the available bandwidth and transmission power that will be provided for the offloading user. Then the UE's current serving BS calculates the throughput, that each bidder can provide, to decide whether it could satisfy the UE's QoS requirement.

The target BS for offloading will be the winner of the reverse auction process. The conditions for judging the winner bid could be set as the optimal objectives for the offloading process. In this paper, the optimal objective of the proposed reverse auction based GO scheme is to maximize the system energy efficiency subjected to the UE's minimum throughput requirement and bidder's available resources during the offloading process. The reverse auction based GO scheme involves two steps: Allocation and Pricing.

In the Allocation step, the UE's current serving BS decides which bidders will be the auction winners. As described above, the coordination techniques among different BSs are supported in the offloading scheme. Therefore, the winner may be one BS or several BSs with the same bids.

In the Pricing step, the improvement of system energy efficiency is evaluated as a payment from the UE. Finally, the UE's current serving BS sends back the auction result to the bidders which consist of the required resources and the expected energy efficiency increment. Then, the handover process for the offloading will start. As shown in Figure 1, 
the winning bidders are BS1 and BS3 together, who win the reverse auction and are designated as the offloading target BSs to serve the UE coordinately.

In order to present the reverse auction based GO scheme clearer, the related notation definitions are introduced as follows:

Bid $\left(b_{i}\right)$ : submitted by the $B S_{i}$ to convey how much bandwidth and transmission power it can provide to the offloading UE, which may not always equal all available resources the BS can provide.

Private Value $\left(x_{i}\right)$ : available resources in the $B S_{i}$, which is only known by the $B S_{i}$ itself.

Pricing $\left(p_{i}\right)$ : the opportunity cost of the $i$ th BS, which makes sure the system energy efficiency could be maximum.

Based on the auction theory, when the condition $b_{i}=x_{i}$ is satisfied, the auction process is truthful. Moreover, $b_{i}=$ $x_{i}$ is a weakly dominant strategy [20]. As a result, in this paper, we set $b_{i}=x_{i}$, assuming all the bidders participating in the auction process will send the bids with all available resources it can provide, which guarantees the truthfulness of the reverse auction model.

Although we have set the Bid equal to the PrivateValue, the reverse auction based GO scheme with multiple candidate target BSs is still NP-hard problem. In order to solve this problem, an approximation algorithm is designed in the next section. The notations used in the algorithm are introduced in the Notation Definition.

2.3. User Delay Tolerance. The reverse auction based GO scheme will start upon the receipt of offloading request at the serving BS periodically. For the traditional auction process, there usually exist multiround bidding procedures to achieve the final winner. This process would inevitably generate an extra delay for the BSs to wait for the auction consequence. But for the wireless network, the user traffic usually has delay tolerance requirements, which should be considered in the offloading scheme. Therefore, we implement the single-round auction in this paper, in order to prevent the information exchange overhead and corresponding delay for the offloading users. Since the number of BSs surrounding a specific UE is limited, the extra delay caused by the singleround first price sealed bid auction process could be not significant. This delay avoidance mechanism is also feasible for the handover based offloading schemes that usually need more time for measurements and handover decisions.

\section{Reverse Auction Based GO Scheme}

In this section, the proposed reverse auction based GO scheme is described. The main steps of the proposed GO scheme are given in Figure 1. Firstly, according to the offloading request, the UE's current serving BS collects all the bids from its neighboring BSs. Then the serving BS calculates the throughput that all bidders can provide and derives the expected energy efficiency increment for each candidate BS. Based on the derived throughput and energy efficiency increment, a single-round reverse auction process is performed, which includes the Allocation and Pricing steps. Finally, the auction results are sent back to the bidders and the user will be offloaded to the winners accordingly.

3.1. Bidding. In order to contribute to the offloading process, bidders will append available resources with their bids to reveal the throughput they can provide for the offloading user. For each bidder, the upper bound of bandwidth and transmission power that a BS can provide is $B_{\text {bound }}$ and $P_{\text {bound }}$, respectively. $B_{\text {bound }}$ and $P_{\text {bound }}$ can be divided into multiple units and classified as multiple bids $\mathbf{b}=\left\{\mathbf{b}^{1}, \mathbf{b}^{2}, \ldots, \mathbf{b}^{i}, \ldots, \mathbf{b}^{l}\right\}$ to indicate the resources the UE can obtain from each bidder, where $l=\max \left(\left\lfloor B_{\text {bound }} / e_{B}\right\rfloor,\left\lfloor P_{\text {bound }} / e_{P}\right\rfloor\right) . e_{B}$ is the basic bandwidth unit; $e_{P}$ is the basic transmission power unit for the bidder. The $\mathbf{b}^{i}=\left(B^{i}, P^{i}\right)$ consists of both bandwidth and transmission power resources. After receiving all of the bids, UE's current serving BS can know how many resources each bidder can provide with the value not larger than the $\sum_{i=1}^{l} B^{i}$ and $\sum_{i=1}^{l} P^{i}$. The scale of the bandwidth and transmission power unit can be flexibly set by the system. The smaller unit definition leads to more information in the bid, which improves the performance of the auction process. But it will also generate more computational costs and increase the complexity. In this paper, one Resource Block (RB) and $0.1 \mathrm{~W}$ transmission power are chosen as the basic bandwidth unit and transmission power unit, respectively. We choose $0.1 \mathrm{~W}$ as the basic transmission power here because the Dynamic Programming will be adopted in the next subsection to solve the optimization problem, where the integer data are needed by the Dynamic Programming method.

3.2. Reserve Auction Algorithm. As mentioned above, the reverse auction process includes two steps of the Allocation and Pricing.

3.2.1. Allocation. In traditional reverse auction processes, the allocation result is completely decided by the bids; that is, the bidders who offer the largest supply of resources will win the auction. However, in this paper, besides the resources that the bidders can provide, the energy efficiency achieved by the bidder should also be considered. Assume that $\mathbf{B}=$ $\left\{\mathbf{B}_{1}, \mathbf{B}_{2}, \ldots, \mathbf{B}_{j}, \ldots, \mathbf{B}_{n}\right\}$ and $\mathbf{P}=\left\{\mathbf{P}_{1}, \mathbf{P}_{2}, \ldots, \mathbf{P}_{j}, \ldots, \mathbf{P}_{n}\right\}$ represent the allocation result, where $\mathbf{B}_{j}=\left\{B_{j}^{1}, B_{j}^{2}, \ldots, B_{j}^{l_{j}}\right\}$ is the RB that $j$ th BS could provide and $B_{j}^{i}=0$ if the $i$ th $\mathrm{RB}$ in the $j$ th BS is not needed. $\mathbf{P}_{j}=\left\{P_{j}^{1}, P_{j}^{2}, \ldots, P_{j}^{l_{j}}\right\}$ denotes the transmission power that the $j$ th BS could transmit and $P_{j}^{i}$ is the transmission power on $B_{j}^{i}$. If $\mathbf{B}_{j}$ or $\mathbf{P}_{j}$ equals zero, the $j$ th BS loses in this auction process.

The Allocation problem is formulated as

$$
\begin{aligned}
\max _{\mathbf{B}_{j}, \mathbf{P}_{j}} & \frac{C_{\text {system }}}{P_{t}} \\
\text { s.t. } & \sum_{j=1}^{n} \sum_{i=1}^{l_{j}} B_{j}^{i} \log \left(1+\frac{P_{j}^{i} H_{j}}{I_{j}^{i}+N_{0} B_{j}^{i}}\right) \geq \tau_{r} \quad \forall r
\end{aligned}
$$




$$
\begin{aligned}
& B_{j}^{i} \in\{0, B\} \\
& P_{j}^{i} \in\left\{\mathbf{P}_{m}, \mathbf{P}_{s}\right\} \\
& \mathbf{P}_{m}=\left\{P_{j}^{i} \mid 0 \leq P_{j}^{i} \leq P_{m}, \sum_{i=1}^{l_{j}} P_{j}^{i} \leq P_{m}\right\} \\
& \mathbf{P}_{s}=\left\{P_{j}^{i} \mid 0 \leq P_{j}^{i} \leq P_{s}, \sum_{i=1}^{l_{j}} P_{j}^{i} \leq P_{s}\right\} .
\end{aligned}
$$

In (1), $C_{\text {system }}=\sum_{j=1}^{n} C_{j} \sum_{i=1}^{l_{j}} B_{j}^{i}$ denotes the throughput; $P_{t}=\sum_{i=1}^{l_{j}} P_{j}^{i} \sum_{j=1}^{n} l_{j}$ denotes the expectation of transmission power on each RB from all the bidders. $C_{\text {system }} / P_{t}$ is the system energy efficiency and $P_{t}>0$.

In (2), $H_{j}=d_{j}^{-\alpha_{j}}\left|h_{j}\right|^{2} \chi_{j}$ is the channel gain between the $j$ th BS and UE, where $d_{j}$ is the distance between the $j$ th BS and UE. $\alpha_{j}$ is the path-loss exponent of the $j$ th BS. $h_{j}$ is the Rayleigh fading component. $\chi_{j}$ denotes the log-normally distributed shadow fading. Furthermore, $I_{j}^{i}=$ $\sum_{j^{\prime}=1, j^{\prime} \neq j}^{n} P_{j^{\prime}}^{i} H_{j^{\prime}} v_{j^{\prime}}^{i}$ is the interference experienced by the UE on the $i$ th $\mathrm{RB}$, where $P_{j^{\prime}}^{i}$ is the transmission power on the $i$ th $\mathrm{RB}$ from the $j^{\prime}$ th BS. $H_{j^{\prime}}$ is the channel gain between the $j^{\prime}$ th BS and UE. $v_{j^{\prime}}^{i}$ is the binary variables representing the activity factor of $j^{\prime}$ th BS. $N_{0}$ is the thermal noise level and $\tau_{r}$ is the guaranteed throughput threshold of the offloading UE.

Constraint (3) means the RB $B_{j}^{i}$ can be occupied or vacant. Constraint (4) denotes the bidder can be a macrocell BS or a small cell BS. In (5) and (6), we give the requirement for the transmission power in the corresponding RB, where $P_{m}$ and $P_{s}$ are the power limitations for the macrocell BS and small cell BS, respectively.

For the convenience of solving this problem, we transform (1), (2), and (3) into the following form:

$$
\begin{aligned}
\min _{\mathbf{B}_{j}, \mathbf{P}_{j}} & \frac{P_{t}}{C_{\text {system }}} \\
& C_{\text {system }} \geq \tau_{r} \\
& 0 \leq B_{j}^{i} \leq B .
\end{aligned}
$$

Assuming the $C_{\text {system }}$ is an independent variable, the Karush-Kuhn-Tucker (KKT) conditions are given as

$$
\begin{gathered}
\nabla_{\mathbf{B}_{j}, \mathbf{P}_{j}, \text { system }} \frac{P_{t}}{C_{\text {system }}}-\nabla_{\mathbf{B}_{j}, \mathbf{P}_{j}, \text { system }}\left(C_{\text {system }}-\tau_{r}\right) \mu \\
-\nabla_{\mathbf{B}_{j}, \mathbf{P}_{j}, \text { system }} B_{j}^{i} \sigma_{j}^{i}-\nabla_{\mathbf{B}_{j}, \mathbf{P}_{j}, \text { system }}\left(B-B_{j}^{i}\right) \xi_{j}^{i} \\
-\nabla_{\mathbf{B}_{j}, \mathbf{P}_{j}, \text { system }} P_{j}^{i} v_{j}^{i}-\nabla_{\mathbf{B}_{j}, \mathbf{P}_{j}, \text { system }}\left(P_{s}\left(P_{m}\right)-P_{j}^{i}\right) \lambda_{j}^{i} \\
-\nabla_{\mathbf{B}_{j}, \mathbf{P}_{j}, \text { system }}\left(P_{s}\left(P_{m}\right)-\sum_{i=1}^{l_{j}} P_{j}^{i}\right) \rho_{j}=0
\end{gathered}
$$

$$
\begin{aligned}
& \left(C_{\text {system }}-\tau_{r}\right) \mu=0 \\
& B_{j}^{i} \sigma_{j}^{i}=0, \quad B_{j}^{i} \geq 0, \quad \sigma_{j}^{i} \geq 0 \\
& \left(B-B_{j}^{i}\right) \xi_{j}^{i}=0, \quad B \leq B_{j}^{i}, \quad \xi_{j}^{i} \geq 0 \\
& P_{j}^{i} v_{j}^{i}=0, \quad P_{j}^{i} \geq 0, \quad \nu_{j}^{i} \geq 0 \\
& \left(P_{s}\left(P_{m}\right)-P_{j}^{i}\right) \lambda_{j}^{i}=0, \quad P_{j}^{i} \leq P_{s}\left(P_{m}\right), \quad \lambda_{j}^{i} \geq 0 \\
& \left(P_{s}\left(P_{m}\right)-\sum_{i=1}^{l_{j}} P_{j}^{i}\right) \rho_{j}=0, \quad \sum_{i=1}^{l_{j}} P_{j}^{i} \leq P_{s}\left(P_{m}\right), \rho_{j} \geq 0 .
\end{aligned}
$$

Equation (8) is changed into (15) by taking the derivative of $C_{\text {system }}$ as

$$
\begin{gathered}
-\frac{P_{t}}{C_{\text {system }}^{2}}-\mu=0 \\
\frac{P_{t}}{C_{\text {system }}^{2}}+\mu=0 .
\end{gathered}
$$

This makes sense, where $P_{t}$ and $C_{\text {system }}^{2}$ are nonzero values. Thus, $\mu \neq 0$, (9) shows $\left(C_{\text {system }}-\tau_{r}\right) \mu=0$, where

$$
C_{\text {system }}=\tau_{r} \text {. }
$$

When the offloading process is triggered, the offloaded user traffic requirements and the number of BSs participating in the auction are known. The KKT conditions prove that (9) is guaranteed throughput requirement. The Allocation problem is transformed to solve $\min _{\mathbf{B}_{j}, \mathbf{P}_{j}} P_{t}$, which can be simplified into a linear objective function $\min \sum_{i=1}^{l_{j}} P_{j}^{i} \sum_{j=1}^{n} l_{j}$.

Because the above Allocation problem is a linear problem, it is easy to find that this is a multiple knapsack problem. In order to facilitate the solving process, we turn it into a 0-1 knapsack problem. There are $n$ BSs to participate in the auction; the $i$ th BS resources are separated into $M_{i}$ independent piece of "goods" that can be loaded into a knapsack. This will get a 0-1 knapsack problem where the number of items is $\sum M_{i}$. If we solve this problem directly, the computational complexity will be $O\left(C \sum M_{i}\right)$. In order to reduce the complexity, we design another algorithm with the specific plan as follows.

As mentioned above, the $i$ th BS bandwidth resource is composed of a number of RB groups. Considering the condition of the binary, the guaranteeing of selecting any multiple resource package strategy still can be achieved after the transformation of the original multiple knapsack problem. One BS which has $M_{i}$ resource blocks is separated into several RB groups, where these RB groups, respectively, have $1,2,2^{2}, 2^{3}, \ldots, M_{i}-2^{k-1}+1$ resource blocks. The $i$ th BS has Ceiling $\left(\log M_{i}\right)$ different RB groups participating in the 0-1 knapsack problem.

Initialize the value as follows: the weight of knapsack is $C$, which is the offloading user traffic requirements $\tau_{r}$. The starting value $F[0, c]=0, F[x, 0]=0$. And the $i$ th $\mathrm{BS}$ has Ceiling $\left(\log M_{i}\right)$ stages. 


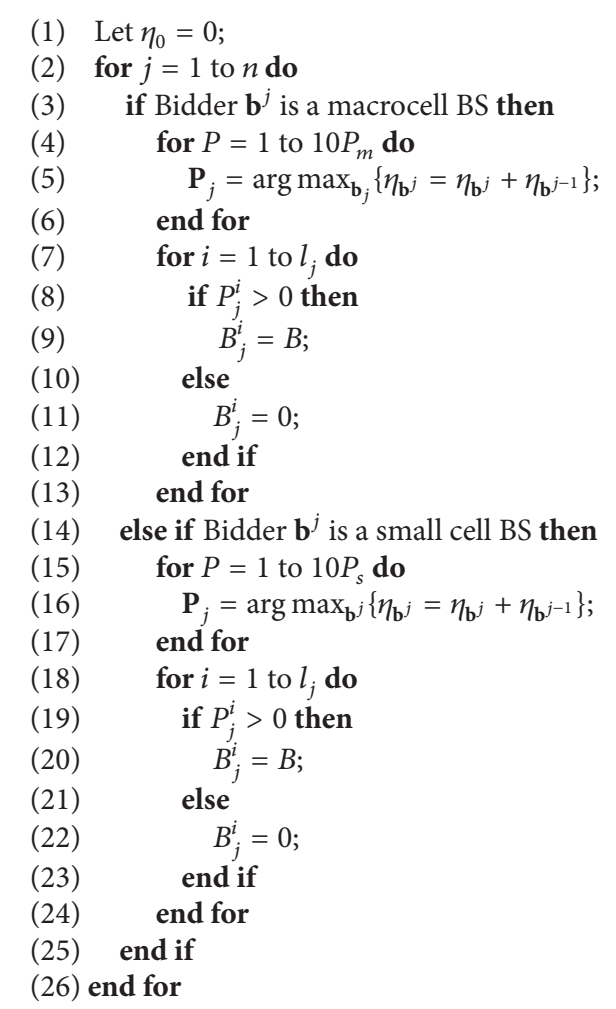

Algorithm 1: Reverse auction, Allocation $\left(n, \mathbf{b}_{n}\right)$.

Renumber all RB groups, $c_{x}$ is the capacity of the $x$ stage, which denotes the $i$ th RB group of the $j$ th BS, and the corresponding value is $v_{x}$.

The capacity of the $x$ stage is

$$
c_{x}=w[i, j]=B_{j}^{i} \log \left(1+\frac{P_{j}^{i} H_{j}}{I_{j}^{i}+N_{0} B_{j}^{i}}\right) .
$$

The value of the corresponding $v_{x}$ is

$$
v_{x}=v[i, j]=P_{j}^{i}
$$

The iterative equation will be

$$
F[x, c]=\min \left\{F[x-1, c], F\left[x-1, c-c_{x}\right]+v_{x}\right\} .
$$

In (20), $F[x, c]$ is the minimum value of the transmission power in the stage $x . c$ denotes the remaining space of the pack according to the current stage. $c_{x}$ denotes the provided capacity when choosing the $x$ stage. $v_{x}$ denotes the provided transmission power when choosing the $x$ stage. The computational complexity is reduced to $O\left(C \sum \log M_{i}\right)$.

The proposed Allocation algorithm is illustrated in Algorithm 1 with $\mathbf{B}=\left\{\mathbf{B}_{1}, \mathbf{B}_{2}, \ldots, \mathbf{B}_{n}\right\}$ and $\mathbf{P}=\left\{\mathbf{P}_{1}, \mathbf{P}_{2}, \ldots\right.$, $\left.\mathbf{P}_{n}\right\}$.

In Algorithm 1, the transmission power of each bidder is chosen firstly and the corresponding bandwidth is decided based on the transmission power allocation results. As

\author{
for $j=1$ to $n$ do \\ (2) if $j$ th BS is a winning bidder then \\ Reverse Auction - Allocation $\left(n \backslash\{j\}, \mathbf{B} \backslash\left\{\mathbf{b}^{i}\right\}\right)$ \\ (4) $\quad p_{j}=\eta_{\mathbf{B}_{n} \backslash\left\{\mathbf{b}^{i}\right\}}-\left(\eta_{\mathbf{B}_{n}}-\eta_{\mathbf{b}^{i}}\right)$ \\ else \\ (6) $\quad p_{j}=0$; \\ end if \\ (8) end for
}

Algorithm 2: Reverse auction, Pricing $\left(n, \mathbf{b}_{n}, \mathbf{B}, \mathbf{P}, \eta\right)$.

mentioned before, $0.1 \mathrm{~W}$ is chosen as the basic transmission power unit and the integer data is needed in the Dynamic Programming. Therefore, the range of $P$ is defined from 1 to $10 P_{m}$ or $10 P_{s}$. The equation in Line (5) and Line (16) of Algorithm 1 mean that the $j$ th bidder's transmission power should be chosen to achieve the largest $\eta_{\mathbf{b}^{j}}$ and guarantee the offloaded user's throughput threshold $\tau_{r}$ at the same time, where $\eta_{\mathbf{b}^{j}}$ denotes the energy efficiency of the $j$ th bidder. After implementing the Dynamic Programming, the results of Algorithm 1, P/10 and B, will be the optimal allocation solution for the proposed reverse auction process.

3.2.2. Pricing. In traditional Pricing algorithms, the bidders are encouraged to set their own bids truthfully, as illustrated before. So, in this paper, the same energy efficiency that the corresponding bidder achieves is paid back. With regard to the offloading user throughput threshold $\tau_{r}$, we define $\eta_{1}$ and $\eta_{2}$ as (21) and (22) as follows:

$$
\begin{aligned}
& \eta_{1}=\eta_{\mathbf{B}_{n} \backslash\left\{\mathbf{b}^{i}\right\}}=\max _{\mathbf{B}_{j} \backslash\left\{\mathbf{B}_{i}\right\}, \mathbf{P}_{j} \backslash\left\{\mathbf{P}_{i}\right\}} \frac{C_{\text {system }}}{E\left(P_{t}\right)} \\
& \eta_{2}=\eta_{\mathbf{B}_{n}}-\eta_{\mathbf{b}^{i}}=\left(\max _{\mathbf{B}_{j}, \mathbf{P}_{j}} \frac{C_{\text {system }}}{E\left(P_{t}\right)}\right)-\frac{C_{\mathbf{b}^{i}}}{E\left(\mathbf{P}_{i}\right)},
\end{aligned}
$$

where $\eta_{1}$ denotes the system energy efficiency under the optimal Allocation solution without the presence of the $i$ th BS. The $\eta_{2}$ denotes the system energy efficiency except for the $i$ th BS under current optimal Allocation results. Then the opportunity cost of the $i$ th BS is defined as the difference between $\eta_{1}$ and $\eta_{2}$, just as illustrated in (23) [19] as follows:

$$
p_{i}=\eta_{1}-\eta_{2}=\eta_{\mathbf{B}_{n} \backslash\left\{\mathbf{b}^{i}\right\}}-\left(\eta_{\mathbf{B}_{n}}-\eta_{\mathbf{b}^{i}}\right) .
$$

The Pricing algorithm is given as Algorithm 2.

3.2.3. Properties. In this section, the properties of the proposed reverse auction model are analyzed. According to the VCG based reverse auction model, the IndividualRationality and the Truthfulness properties need to be proved.

IndividualRationality. When the utility of each participating bidder in the Pricing stage is greater than zero, this algorithm is individual rational for each winning bidder. Namely,

$$
p_{i}=\eta_{\mathbf{B}_{n} \backslash\left\{\mathbf{b}^{i}\right\}}-\left(\eta_{\mathbf{B}_{n}}-\eta_{\mathbf{b}^{i}}\right) \geq 0 .
$$


Truthfulness. For each bidder, the Truthfulness means that each bidder's bid price is equal to its private value. This is a weakly dominant strategy. If BS's bidding is untrue, the energy efficiency will be unlikely the biggest. In order to get the maximum energy efficiency, the allocation should be formulated as follows:

$$
\begin{aligned}
p_{j} & =\eta_{\mathbf{B}_{n} \backslash\left\{\mathbf{b}^{j}\right\}}-\left(\eta_{\mathbf{B}_{n}}-\eta_{\mathbf{b}^{j}}\right) \\
\delta & =p_{j}-p_{i} \\
& =\eta_{\mathbf{B}_{n} \backslash\left\{\mathbf{b}^{j}\right\}}-\left(\eta_{\mathbf{B}_{n}}-\eta_{\mathbf{b}^{j}}\right)-\left[\eta_{\mathbf{B}_{n} \backslash\left\{\mathbf{b}^{i}\right\}}-\left(\eta_{\mathbf{B}_{n}}-\eta_{\mathbf{b}^{i}}\right)\right] \\
& =\eta_{\mathbf{B}_{n} \backslash\left\{\mathbf{b}^{j}\right\}}-\eta_{\mathbf{B}_{n}}+\eta_{\mathbf{b}^{j}}-\eta_{\mathbf{B}_{n} \backslash\left\{\mathbf{b}^{i}\right\}}+\eta_{\mathbf{B}_{n}}-\eta_{\mathbf{b}^{i}} \\
& =\eta_{\mathbf{B}_{n} \backslash\left\{\mathbf{b}^{j}\right\}}+\eta_{\mathbf{b}^{j}}-\eta_{\mathbf{B}_{n} \backslash\left\{\mathbf{b}^{i}\right\}}-\eta_{\mathbf{b}^{i}} \\
& =\left(\eta_{\mathbf{B}_{n} \backslash\left\{\mathbf{b}^{j}\right\}}+\eta_{\mathbf{b}^{j}}\right)-\left(\eta_{\mathbf{B}_{n} \backslash\left\{\mathbf{b}^{i}\right\}}+\eta_{\mathbf{b}^{i}}\right) .
\end{aligned}
$$

Based on the proposed model in this paper, because $p_{j} \leq$ $p_{i}$ and $\delta \leq 0$, this means $\eta_{\mathbf{B}_{\eta} \backslash\left\{\mathbf{b}^{j}\right\}}+\eta_{\mathbf{b}^{j}} \leq \eta_{\mathbf{B}_{n} \backslash\left\{\mathbf{b}^{i}\right\}}+\eta_{\mathbf{b}^{i}}$. If and only if $j=i$, it can take the equal sign. Therefore, each bidder must be truthful to obtain the maximum system energy efficiency. The proof is finished.

\section{Performance Evaluation}

In this section, we built the system-level simulation platform according to the 3GPP LTE-Advanced simulation methodology [23]. Based on this platform, we validate the performances of the proposed reverse auction based GO scheme with comparison algorithms in the small cell HetNet downlink scenario.

\subsection{Simulation Setting, Performance Metrics, and Comparison} Algorithms. The considered simulation scenario in this paper comprises 19-hexagonal macrocells with 3 sectors per macrocell. In each sector, there is one small cell cluster deployed with shared spectrum manner. The small cell cluster is a group of densely deployed small cells. We deploy $2 / 3$ users in the coverage of small cell clusters, while the remaining users are distributed in the coverage area of macro cells. The users are uniformly distributed. Moreover, as mentioned above, the bandwidth resource granularity in the simulation is one RB. In the initial state, each user is served by the BS which can provide the highest downlink RSRP. Once a new traffic offloading requirement is requested, the reverse auction based GO scheme is triggered. The detailed simulation parameters are according to 3GPP LTE-Advanced small cell HetNet evaluation methodology [24]. These parameters are listed in Table 1.

The performance metrics include the system energy efficiency, offloading gain, and throughput. In this paper, the metric of offloading gain $\left(\gamma_{\text {gain }}\right)$ is defined as

$$
\gamma_{\text {gain }}=\frac{\tau_{\text {offloading }}}{\tau_{\text {total }}}
$$

where $\tau_{\text {offloading }}$ denotes the offloaded throughput and $\tau_{\text {total }}$ denotes the total system throughput. The offloading gain is
TABLE 1: Simulation parameters.

\begin{tabular}{lc}
\hline Simulation parameter & Value \\
\hline Carrier frequency & $2 \mathrm{GHz}$ \\
System bandwidth & $10 \mathrm{MHz}$ \\
Total transmission power of & $46 \mathrm{dBm}$ \\
macrocell & $30 \mathrm{dBm}$ \\
Total transmission power of small cell & $\mathrm{Pl}=28.3+22.0 \log _{10}(d)$ \\
Path-loss of macrocell & $\mathrm{Pl}=30.5+36.7 \log _{10}(d)$ \\
Path-loss of small cell & $4 \sim 10$ \\
Small cell number per cluster & 3 \\
Small cell cluster number per & 60 \\
macrocell & $17 \mathrm{dBi}$ \\
User number per macrocell & $5 \mathrm{dBi}$ \\
Antenna gain of macrocells & FTP Model 1 \\
Antenna gain of small cells & $-174 \mathrm{dBm} / \mathrm{Hz}$ \\
Traffic model & $5 \mathrm{Mbps}$ \\
Power spectrum density of thermal & \\
noise & User throughput threshold
\end{tabular}

a more straightforward notation about how much traffic load could be offloaded to improve the energy efficiency.

In order to evaluate the performances of the proposed $\mathrm{GO}$ scheme, we compare it with the TOFFR algorithm proposed in [13] and the incentivized scheme proposed in [14], which have been introduced in the related works. The simulation results are given as below.

4.2. Impacts of Small Cell Numbers on Energy Efficiency. According to 3GPP simulation assumptions, there are $2 / 3$ UEs distributed in the coverage of small cell cluster, while the remaining UEs are uniformly distributed in the remaining area of macrocells. In this section, the impacts of deployment density of small cells in a cluster on the system energy efficiency are investigated. According to 3GPP simulation methodology, the number of small cells in a cluster varies from 4 to 10 .

As shown in Figure 2, the system energy efficiency of different algorithms versus small cell numbers per cluster is demonstrated. We can observe that the system energy efficiency is improved with the increasing of small cell numbers. The reason lies that small cells usually can provide higher energy efficiency than macrocells due to lower transmission power attenuations in hot spot deployment scenarios. When there exist more small cells inside one macrocell, more user traffic could be offloaded potentially to small cells. Therefore, higher system energy efficiency could be further achieved.

But the system energy efficiency increases slightly when the number of small cells is large. This is because when the small cells are deployed more densely, the intercell interferences will be more severe, indicating the requirement of larger transmission power to ensure the same user throughput. Moreover, from results in Figure 2, we have proved that the proposed GO scheme outperforms the TOFFR algorithm and incentivized scheme regardless of small cell density deployed 


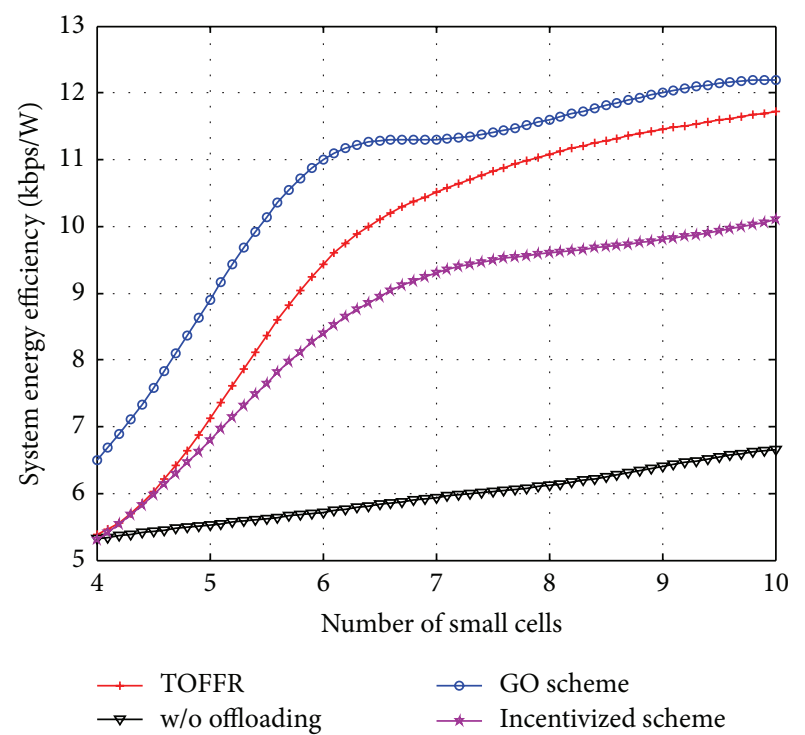

FIGURE 2: System energy efficiency versus small cell numbers per cluster.

per cluster. In the TOFFR algorithm, the fractional frequency reuse scheme is adopted to improve UE performances better in the cell edge. The UE located in the central area cannot contribute to the energy efficiency improvement. The incentivized scheme focuses on maximizing the offloading utility that purchases the available unused bandwidth in femtocells. So, the proposed GO scheme has better energy efficiency performance than both of them.

We also compare these three offloading algorithms with the situation of no offloading (denoted as w/o offloading in simulations) as the baseline. It is obvious that when offloading schemes are adopted, more user traffic originally served by macrocell will be actively offloaded to small cells. Therefore, all of the offloading schemes, including the proposed GO scheme, TOFFR algorithm, and incentivized algorithm, achieve higher system energy efficiency.

4.3. Impacts of Small Cell Numbers on Offloading Gain. As shown in Figure 3, the offloading gain versus different small cell numbers per cluster is dipicted. We can observe that the offloading gain increases with small cell numbers, due to the capacity growth with the increase of small cell numbers per cluster. Besides, in order to maximize the system energy efficiency, there is limitation on the amount of offloaded throughputs, as demonstrated in Figure 3. When the offloading gain reaches $62 \%$, the rise against the small cell numbers becomes rather slow. The simulation results further suggest that the proposed GO scheme outperforms the TOFFR algorithm and incentivized scheme in terms of not only energy efficiency, but also the offloading gain, because in TOFFR algorithm and incentivized scheme the offloading is mainly focused on the cell edge users, which limits the performance improvements. Moreover, Figure 3 shows that the increasing of the small cell numbers of all these three schemes will reach a plateau. The reason lies that there are always several specific users out of the coverage of the small

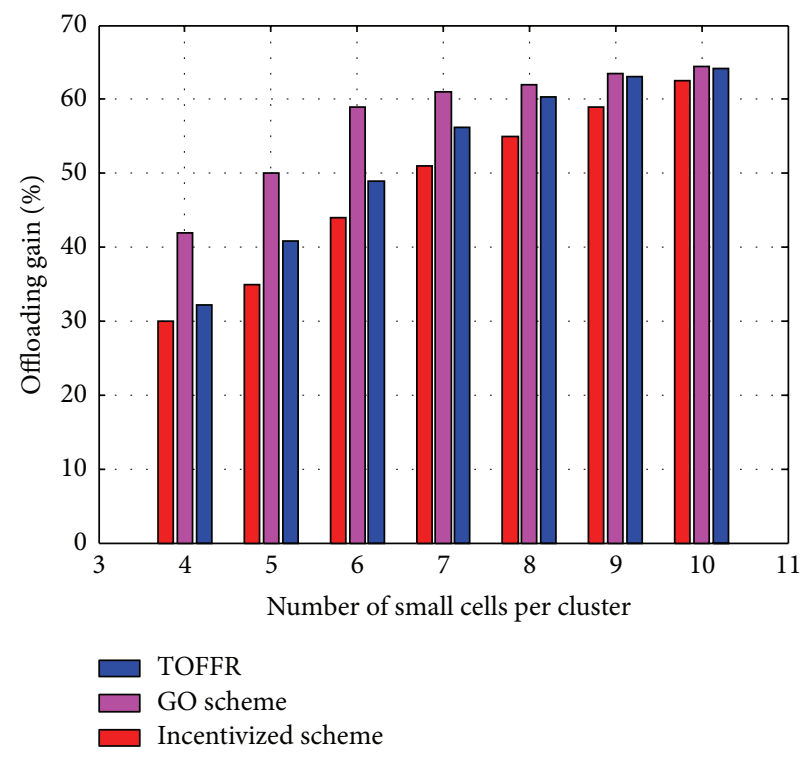

FIGURE 3: Offloading gain versus small cell numbers per cluster.

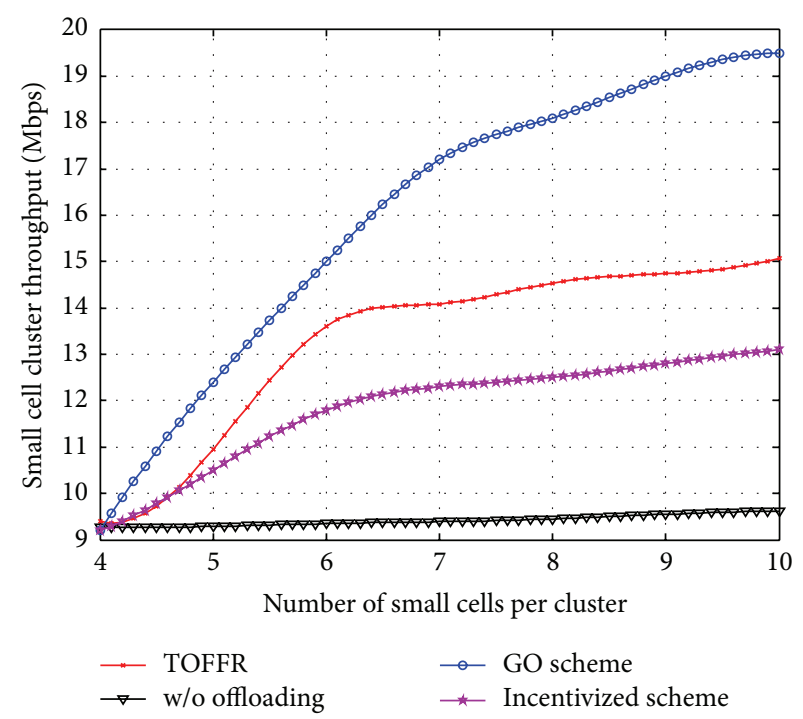

FIGURE 4: Small cell throughput versus small cell numbers per cluster.

cell clusters in the simulations. Just as mentioned before, $2 / 3$ UEs are deployed in the coverage of small cell cluster and the simulation results also show that the offloading gain limitation of all three algorithms can only reach to near $2 / 3$, which in turn indicates the offloading limitation will be decided by the distribution and location of users.

4.4. Impact of Small Cell Numbers on Small Cell Throughput. In this section, the impacts on the throughput of small cell cluster versus different small cell numbers are investigated. From Figure 4, we can see the throughput increases with the increase of small cell numbers per cluster. The reason is that more users will be served by the small cells after offloading processes. Moreover, the throughput increments grow slowly 
when small cell numbers per cluster are relatively large. This is because of the increase of intercell interferences caused by denser small cell deployments. Finally, the results in Figure 4 prove that the proposed GO scheme outperforms TOFFR algorithm and incentivized algorithm also in terms of small cell cluster throughput.

\section{Conclusion}

This paper aims to solve the problem regarding how to perform offloading in the small cell HetNet deployments with optimization on maximizing the system energy efficiency. The reverse auction theory has been implemented with the proposed GO scheme design to decide the offloading target BS or BSs with coordination transmission enabled technology. The reverse auction model is formulated by multiple sellers (BSs) and a single buyer (offloading user) with the first price sealed bid mechanism. The BS coordination transmissions are also supported for multiple winning bidders scenarios. According to the proposed reverse auction based GO scheme, the energy efficiency optimization problem with constraints of user guaranteed throughput threshold, bandwidth occupation, and transmission power limitation is solved by Dynamic Programming method with KKT conditions. The Individual Rationality and Truthfulness of the VCG based reverse auction model are also proved in the paper. System-level simulations have been conducted to verify the effectiveness of the proposed GO scheme according to 3GPP LTE-Advanced evaluation methodologies. The performances when applying the GO scheme, comparison schemes, and the baseline without offloading situation are evaluated with performance metrics of energy efficiency, offloading gain, and throughput. The simulation results prove that the proposed GO scheme can achieve supreme performances.

\section{Notation Definition}

$\mathbf{b}=\left\{\mathbf{b}^{1}, \mathbf{b}^{2}, \ldots, \mathbf{b}^{i}, \ldots, \mathbf{b}^{l}\right\}: \quad$ Bids

$\mathbf{B}=\left\{\mathbf{B}_{1}, \mathbf{B}_{2}, \ldots, \mathbf{B}_{j}, \ldots, \mathbf{B}_{n}\right\}:$ Bandwidth allocation results

$\mathbf{P}=\left\{\mathbf{P}_{1}, \mathbf{P}_{2}, \ldots, \mathbf{P}_{j}, \ldots, \mathbf{P}_{n}\right\}:$ Power allocation results

$\mathbf{B}_{j}=\left\{B_{j}^{1}, B_{j}^{2}, \ldots, B_{j}^{l_{j}}\right\}: \quad j$ th BS bandwidth allocation

$\mathbf{P}_{j}=\left\{P_{j}^{1}, P_{j}^{2}, \ldots, P_{j}^{l_{j}}\right\}:$ results

$\begin{array}{ll}B_{j}^{i}: & i \text { th subcarrier in the } j \text { th BS } \\ P_{j}^{i}: & \text { Transmission power on } B_{j}^{i} \\ \mathbf{b}_{n}=\left\{\mathbf{b}^{1}, \mathbf{b}^{2}, \ldots, \mathbf{b}^{n}\right\}: & \text { Bids sent by first } n \text { BSs } \\ \eta: & \text { Energy efficiency. }\end{array}$

\section{Competing Interests}

The authors declare that they have no competing interests.

\section{Acknowledgments}

This paper is supported by the National High Technology Research and Development Program of China no. 2014AA01A701, Nature and Science Foundation of China under Grants nos. 61471068 and 61421061, International Collaboration Project no. 2015DFT10160, and National Major Project no. 2016ZX03001009-003.

\section{References}

[1] T. Nakamura, S. Nagata, A. Benjebbour et al., "Trends in small cell enhancements in LTE advanced," IEEE Communications Magazine, vol. 51, no. 2, pp. 98-105, 2013.

[2] W. Ni and I. B. Collings, "A new adaptive small-cell architecture," IEEE Journal on Selected Areas in Communications, vol. 31, no. 5, pp. 829-839, 2013.

[3] L. Hanzo, H. Haas, S. Imre, D. O’Brien, M. Rupp, and L. Gyongyosi, "Wireless myths, realities, and futures: from $3 \mathrm{G} / 4 \mathrm{G}$ to optical and quantum wireless," Proceedings of the IEEE, vol. 100, pp. 1853-1888, 2012.

[4] The METIS 2020 Project - Laying the Foundation of 5G, https:// www.metis2020.com/.

[5] "Small cell market status," White Paper, Informa and Small Cell Forum 1, 2013.

[6] D. Calin, H. Claussen, and H. Uzunalioglu, "On femto deployment architectures and macrocell offloading benefits in joint macro-femto deployments," IEEE Communications Magazine, vol. 48 , no. 1 , pp. 26-32, 2010.

[7] S.-I. Sou, "Mobile data offloading with policy and charging control in 3GPP core network," IEEE Transactions on Vehicular Technology, vol. 62, no. 7, pp. 3481-3486, 2013.

[8] J. Korhonen, T. Savolainen, A. Y. Ding, and M. Kojo, "Toward network controlled IP traffic offloading," IEEE Communications Magazine, vol. 51, no. 3, pp. 96-102, 2013.

[9] H. Elsawy, E. Hossain, and S. Camorlinga, "Traffic offloading techniques in two-tier femtocell networks," in Proceedings of the IEEE International Conference on Communications (ICC '13), pp. 6086-6090, IEEE, Budapest, Hungary, June 2013.

[10] Z. Lu, P. Sinha, and R. Srikant, "EasyBid: enabling cellular offloading via small players," in Proceedings of the 33rd IEEE Conference on Computer Communications (IEEE INFOCOM '14), pp. 691-699, IEEE, Toronto, Canada, May 2014.

[11] M. Usman, A. Västberg, and T. Edler, "Energy efficient high capacity HETNET by offloading high QoS users through femto," in Proceedings of the 17th IEEE International Conference on Networks (ICON '11), pp. 19-24, Singapore, December 2011.

[12] P. Chandhar and S. S. Das, "Analytical evaluation of offloading gain in macrocell-femtocell OFDMA networks," in Proceedings of the IEEE 77th Vehicular Technology Conference (VTC Spring '13), pp. 1-6, June 2013.

[13] Q. Liu, G. Feng, and S. Qin, "Energy-efficient traffic offloading in Macro-Pico networks," in Proceedings of the 22nd Wireless and Optical Communications Conference (WOCC '13), pp. 236241, IEEE, Chongqing, China, May 2013.

[14] Y. Jia, M. Zhao, K. Wang, and W. Zhou, "An incentivized offloading mechanism via truthful auction in heterogeneous networks," in Proceedings of the 6th International Conference on Wireless Communications and Signal Processing (WCSP '14), pp. 1-6, Hefei, China, October 2014.

[15] L. Gao, G. Iosifidis, J. Huang, L. Tassiulas, and D. Li, "Bargaining-based mobile data offloading," IEEE Journal on Selected Areas in Communications, vol. 32, no. 6, pp. 1114-1125, 2014.

[16] F. Zhang, W. Zhang, and Q. Ling, "Non-cooperative game for capacity offload," IEEE Transactions on Wireless Communications, vol. 11, no. 4, pp. 1565-1575, 2012. 
[17] X. Xu, H. Zhang, X. Dai, and X. Tao, "Optimal Energy Efficient Offloading in small cell HetNet with auction," in Proceedings of the 9th International Conference on Communications and Networking in China (CHINACOM '14), pp. 335-340, Maoming, China, August 2014.

[18] D. P. Bertsekas, D. A. Castanon, and H. Tsaknakis, "Reverse auction and the solution of inequality constrained assignment problems," SIAM Journal on Optimization, vol. 3, no. 2, pp. 268297, 1993.

[19] M. Khaledi and A. A. Abouzeid, "Dynamic spectrum sharing auction with time-evolving channel qualities," IEEE Transactions on Wireless Communications, vol. 14, no. 11, pp. 5900-5912, 2015.

[20] F. Shen, D. Li, P.-H. Lin, and E. Jorswieck, "Auction based spectrum sharing for hybrid access in macro-femtocell networks under QoS requirements," in Proceedings of the IEEE International Conference on Communications (ICC '15), pp. 3335-3340, IEEE, London, UK, June 2015.

[21] X. Zhuo, W. Gao, G. Cao, and S. Hua, "An incentive framework for cellular traffic offloading," IEEE Transactions on Mobile Computing, vol. 13, no. 3, pp. 541-555, 2014.

[22] W. Vickrey, "Counterspeculation, auctions, and competitive sealed tenders," The Journal of Finance, vol. 16, no. 1, pp. 8-37, 1961.

[23] 3GPP-TR36.814 (v11.1.0), "Evolved Universal Terrestrial Radio Access (E-UTRA); Further advancements for E-UTRA physical layer aspects," 2013.

[24] 3GPP, "Small cell enhancements for E-UTRA and EUTRAN; physical layer aspects, (release 12)," 3GPP TR 36.872, 2013.

[25] 3GPP R1-130744, "WF on evaluation assumptions for SCE physical layer," Huawei, HiSilicon, CATR, CMCC, 2013.

[26] 3GPP, "Study on small cell enhancements for EUTRA and EUTRAN: higher layer aspects,” 3GPP TR 36.842 (v12.0.0), 2013.

[27] 3GPP TS 36.300, “Technical Specification Group Radio Access Network; Evolved Universal Terrestrial Radio Access (EUTRA) and Evolved Universal Terrestrial Radio Access Network (EUTRAN); Overall description; Stage 2 (Release 12)," 2014. 

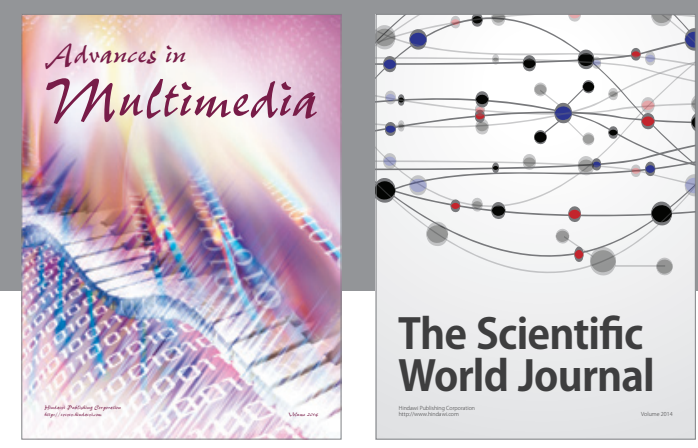

The Scientific World Journal
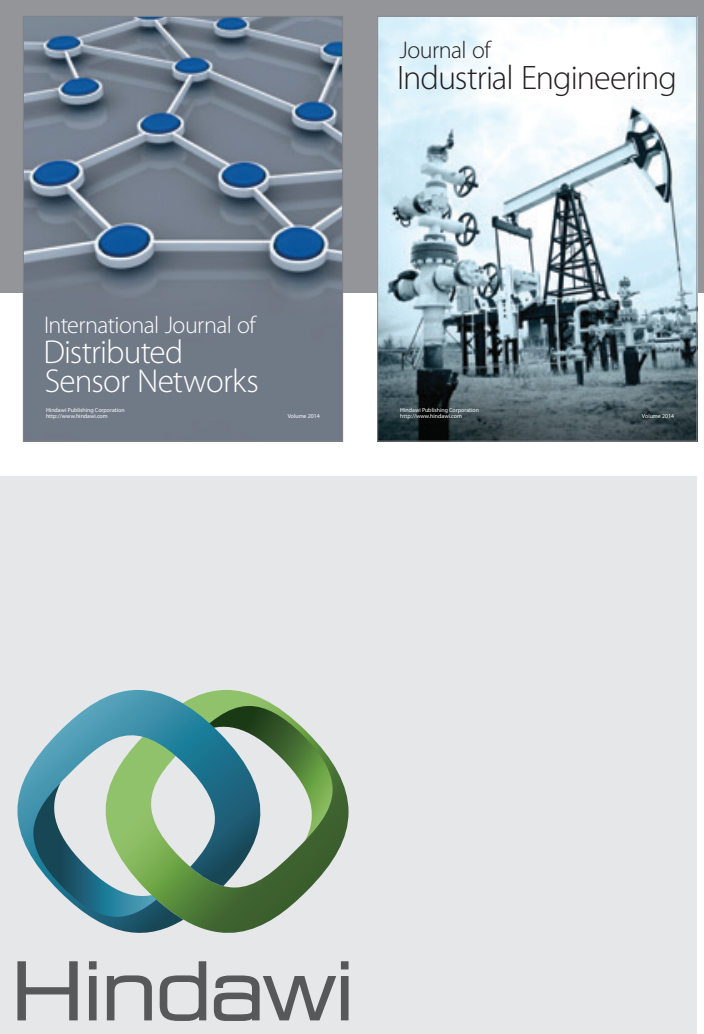

Submit your manuscripts at

http://www.hindawi.com

\section{Computer Networks} and Communications
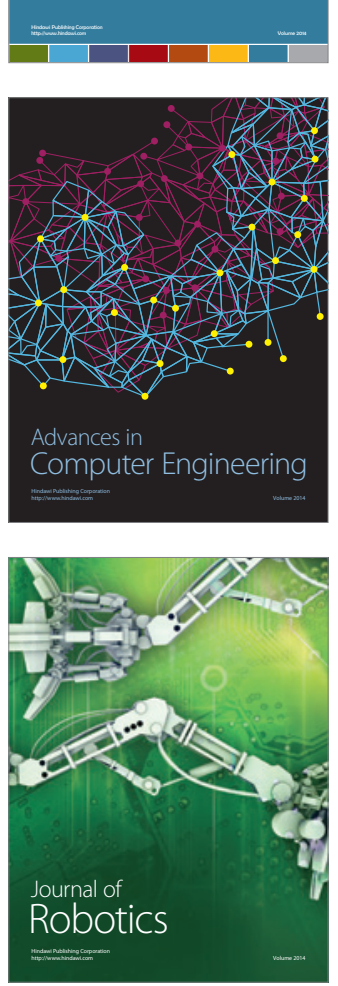
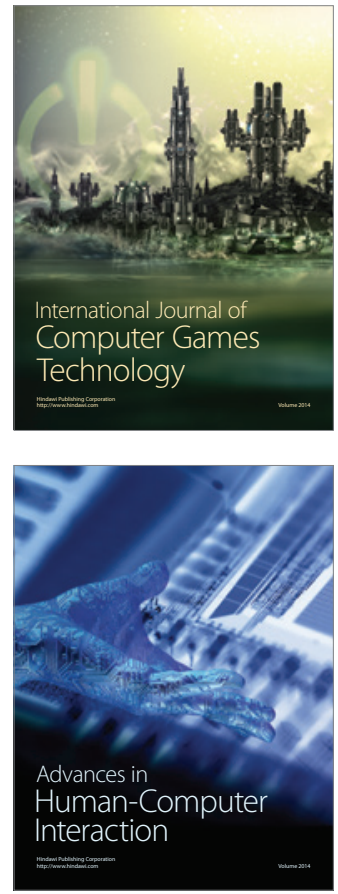
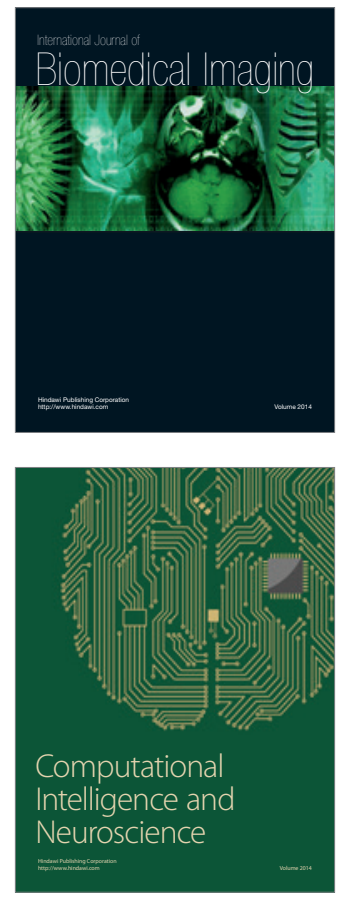
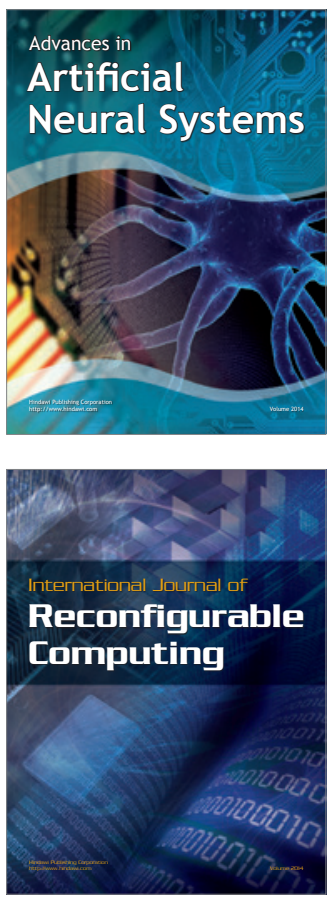
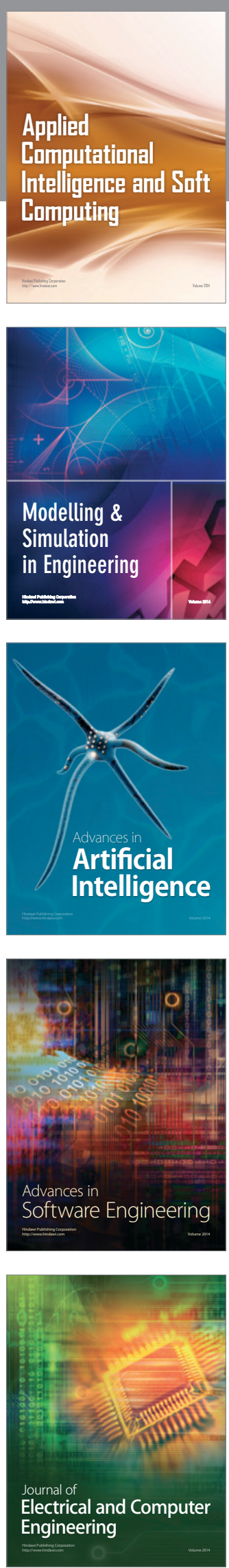